\title{
New Targets for End-Stage Chronic Kidney Disease Therapy
}

\author{
Niki Prakoura, Panos Kavvadas, Christos Chatziantoniou* \\ Inserm UMR S 1155 and University Pierre et Marie Curie, Paris, France
}

\begin{abstract}
Severe forms of chronic kidney disease can lead to a critical, end-stage condition, requiring renal replacement therapy, which may involve a form of dialysis or renal transplantation. Identification and characterization of novel markers and/or targets of therapy that could be applied in these critically ill patients remains the focus of the current research in the field of critical care medicine and has been the objective of our studies for some years past. To this end, we used models of renal vascular disease, Ang II, L-NAME or mice overexpressing renin, treated with AT1 antagonists at different stages of progression, to create cohorts of animals during progression, reversal or escape from therapy. Transcriptomic analysis and comparisons were performed and genes were selected according to the following criteria: a) not previously described in the kidney, b) highly upregulated during progression and returning to the normal levels during reversal, and c) producing proteins that are either circulating or membrane receptors.

The involvement of the selected genes in the mechanisms of renal disease was confirmed in additional models of renal disease, initiated in other compartments of the kidney such as glomeruli (administration of nephrotoxic serum) or the tubular interstitium (unilateral ureteral obstruction). The potential of the therapy was tested using mice lacking the expression of these genes and by in vivo administration of antisense oligonucleotides which blocked the transcription of the targeted genes. This strategy allowed the identification of periostin, an extracellular matrix protein normally involved in bone and tooth development, in addition to the discoidin domain receptor1 (DDR1) as potential targets of therapy against renal inflammation and fibrosis.
\end{abstract}

Keywords: renal fibrosis, renal inflammation, DDR1, periostin

Received: 20 March 2015 / Accepted: 19 June 2015

\section{INTRODUCTION}

Even though significant progress has been made in the identification of the complex mechanisms orchestrating chronic kidney disease $(\mathrm{CKD})$, it remains a fact that there is no specific treatment unequivocally shown to slow or, even better, reverse the progression of renal failure. Severe forms of CKD can lead to a critical, life-threatening condition, requiring renal replacement therapy, which may involve a form of dialysis or renal transplantation [1]. Identification and characterization of novel markers and/or targets of therapy that could be used in the treatment of these critically ill patients represents a priority for current research in the field of critical care medicine. Our laboratory has demonstrated that CKD can be reversed in experimental models by inhibiting the deleterious effects of the renin-angiotensin system [2-4]. However, inhibitors or antagonists of the renin-angiotensin system do not exhibit the same efficiency in arresting or reversing the progression of chronic renal disease in humans [4,5]. Therefore, detection of the disease in early stages and discovery of novel therapeutic targets are of crucial importance for efficient treatment against CKD.

Our team has identified two proteins, periostin and the discoidin domain receptor 1 (DDR1) as promising targets for therapy.

\section{PERIOSTIN}

Periostin is a $90 \mathrm{kDa}$ extracellular protein expressed during development and very early in postnatal tissue $[6,7]$. Periostin expression in healthy adult tissues is low, but increases considerably during certain diseases. A study in asthmatic patients proved that periostin can be a potential read out marker in asthma treat- 
ment [8], while another study involving IPF patients, showed that plasma periostin can be a biomarker for the progression of this disease [9]. Using the model of L-NAME-induced hypertensive nephropathy in rats, we showed that periostin is also over-expressed when renal pathology is present. Interestingly, periostin levels declined when disease was arrested or reversed after treatment with angiotensin receptor 1 blockers, exhibiting a close correlation with the extent of the disease [10]. To strengthen this observation, comparison of periostin levels with creatininemia, proteinuria and renal blood flow showed that periostin correlated inversely to renal function during progression/regression of CKD. These results are in agreement with data from FSGS and Lupus Nephritis patients which indicate that periostin levels correlate well with renal damage and eGFR [11]. Moreover, we have found that mice lacking periostin expression were protected against renal inflammation in the UUO model [12]. This protection was accompanied by a better preservation of the renal tubular epithelium and blunted expression of several pro-fibrotic markers. In addition, delivery of antisense oligonucleotides against periostin protected rats from the progression of renal disease in the L-NAME model [12]. Other investigators showed that periostinis overexpressed in additional experimental models of renal pathology and most importantly, it can be detected in the urine of human patients of $\mathrm{CKD}$, even when albuminuria is within normal values [13].

The precise mechanism through which periostin promotes fibrosis remains largely unknown. Studies in a model of cardiac fibrosis indicated that periostin may act as a downstream mediator of Ang II signaling through complex interactions that involve p38/ CREB and erk/TGF- $\beta$ pathways [14]. Additionally, lung fibroblasts from periostin-/- animals had an impaired response to TNF- $\alpha$, and they failed to produce chemokines and pro-inflammatory cytokines, indicating that periostin could mediate both the inflammatory and the fibrotic process [15].

\section{CAN PERIOSTIN Be A tARget FOR THERA- PY OF CKD?}

Introducing novel biomarkers and therapeutic targets is a complicated, time and money consuming procedure. Several promising candidates have been proven of little value while others, although useful in animal models, are of little use in human pathologies. There- fore, a potential novel biomarker for CKD should be easily detectable in plasma or urine samples, it should be early expressed in the pathology and it should correlate better with the degree of the disease than the existing 'gold standard' biomarkers.

The existing data so far for periostin indicate that:

- Periostin expression is negligible in healthy kidneys.

- Periostin is highly induced in various models of renal disease (UUO, L-NAME, 5/6 nephrectomy, streptozotocin-induced diabetic nephropathy, anti-GBM, renin transgenic mice), and correlates very well with the degree of tissue damage and the decline of renal function in animals.

- Periostin is de novo overexpressed by the cells principally affected in each model and then spreads to other compartments.

- Mice lacking periostin expression are protected against CKD.

- Silencing periostin with anti-sense nucleotides protects animals from L-Name-induced hypertensive nephropathy.

- Periostinis expressed in human biopsies and has been detected in urines from several types of CKD.

\section{DERIOSTIN - CONCLUDING REMARKS}

Several pieces of the puzzle of periostin function in $\mathrm{CKD}$ are missing, especially when it comes to, among other factors, mechanism of action, the downstream and upstream mediators and its paracrine effect on cell populations. However, data so far indicate that at least in experimental models, targeting periostin can result in arrest or even reversal of renal disease while it correlates well with the decline of renal function and renal damage. We are aware that several investigators are currently developing tools to provide, or refute, the proof that periostin has the potential to be a biomarker and a target of therapy of renal disease. We hope that in the near future we will have a definite answer on this hypothesis.

\section{- DISCOIDIN DOMAIN RECEPTOR 1}

DDR1, a member of the tyrosine kinase receptors superfamily, is activated after the binding of extracellular collagen. Its role is well established in adhesion, migration and cell proliferation during physiological development [16]. 
Our team has demonstrated that DDR1 is a candidate effector in renal inflammation and fibrosis. In a model of angiotensin II-induced hypertensive nephropathy, mice lacking DDR1 expression were protected against proteinuria, inflammation, glomerulosclerosis and interstitial fibrosis [17]. In a curative protocol, mice treated with anti-sense oligonucleotides against DDR1 showed preserved renal function and glomerular structure in the anti-GBM model [18]. Data from studies in the UUO model indicated that DDR1 promotes CKD through enhancement of the inflammatory cascade since macrophages from DDR1 KO animals displayed impaired migration in response to MCP1 [19]. In COL4A3 KO mice, a model that mimics the Alport syndrome, deletion of DDR1 delays renal fibrosis via inhibition of NF-kappaB, IL- 6 and TGF- $\beta$ signaling [20].

DDR1's crucial role in the progression of the inflammatory response has been reported in other fibrotic tissues, given the fact that DDR1 KO mice are protected against bleomycin-induced pulmonary fibrosis, exhibiting impaired inflammatory response via deregulation of the p38 MAPK pathway [21].

Regarding human CKD, biopsies from FSGS, diabetic nephropathy and Goodpasture's syndrome, DDR1 exhibited a strong glomerular staining while it was also detectable in parietal epithelial cells, tubules and the interstitium [18].

\section{CAN DDR1 BE A TARGET FOR THERAPY OF CKD?}

The existing data for DDR1 show that:

- DDR1 expression in the healthy kidney is low, but it is over-expressed in several models of renal disease.

- DDR1 amplifies renal injury by enhancing the interaction between renal inflammation and fibrosis.

- Genetic deletion of DDR1 expression impairs the inflammatory response and preserves renal function and structure.

- Silencing of DDR1 gene with anti-sense oligonucleotides preserves renal function.

- DDR1 is over-expressed in several human renal pathologies.

Unpublished data from our laboratory,using the anti-GBM model, further support the hypothesis that DDR1 can be a target for therapy, because DDR1 silencing, after an increase in proteinuria and the estab- lishment of the disease, was accompanied by an arrest in the decline of renal function. Still, several questions regarding the mechanism(s) of action of DDR1 remain unanswered such as the identity of the signal inducing DDR1 neo-expression and/or activation, whether collagens are the only ligands of DDR1, and if DDR1 expression is enough to promote cell phenotype alterations.

Unfortunately, techniques do not yet exist to allow a thorough investigation in human CKD (monoclonal antibodies, Elisa kits, specific blockers). Thus, studies on DDR1's levels in renal biopsies, or correlating its expression with the decline of renal function, are for future research. Hopefully such studies will progress sooner rather than later, and as several groups of investigators are currently pursuing these objectives, the first results are expected in a near future.

\section{CONCLUSIONS: DO PERIOSTIN OR DDR1 HAVE THE POTENTIAL OF TRANSFER IN HU- MAN CKD?}

Our recent investigations, mainly using experimental models, lead us to identify periostin and DDR1 as promising targets of therapythat could be applied, in the future, in critically ill patients with advanced CKD. The observations supporting such a role are: a) both are 'silent' during adulthood under physiological conditions; b) they are de novo activated and their activation is occurring after tissue aggression; c) their expression is focal to the damaged tissue; d) genetic ( $\mathrm{KO}$ mice) orpharmaco-genetic (anti-sense oligonucleotides delivery) of their expression do not affect physiological functions; and e) they are easily accessible for detection and therapy intervention.

The most important limitation is the current lack of widely validated techniquesavailable for use in humans, but this limitation will not exist in a near future as the field is rapidly expanding and several antibodies or blocking agents are expected to be generated or are currently under testing.

\section{口EFERENCES}

1. Lozano, R. Global and regional mortality from 235 causes of death for 20 age groups in 1990 and 2010: a systematic analysis for the Global Burden of Disease Study 2010. Lancet. 2012;380:2095-128.

2. Huby AC, Rastaldi MP, Caron K, Smithies O, Dussaule JC, 
Available online at: www.jccm.ro

Chatziantoniou C. Restoration of podocyte structure and improvement of chronic renal disease in transgenic mice overexpressing renin. PLoS One. 2009;21:e6721.

3. Kavvadas $P$, Weis $L$, Abed AB, Feldman DL, Dussaule JC, Chatziantoniou $C$. Renin inhibition reverses renal disease in transgenic mice by shifting the balance between profibrotic and antifibrotic agents. Hypertension. 2013;61:901-7.

4. Chatziantoniou C, Dussaule JC. Is kidney injury a reversible process? CurrOpinNephrolHypertens. 2008;17:76-81.

5. Mauer M, Zinman B, Gardiner R, et al. Renal and retinal effects of enalapril and losartan in type 1 diabetes. N Engl J Med. 2009;361:40-51.

6. Kruzynska-Frejtag A, Machnicki M, Rogers R, Markwald RR, Conway SJ. Periostin (an osteoblast-specific factor) is expressed within the embryonic mouse heart during valve formation. Mech Dev. 2001;103:183-8.

7. Norris RA, Kern CB, Wessels A, et al.Identification and detection of the periostin gene in cardiac development. AnatRec A Discov Mol CellEvolBiol. 2004;281:1227-33.

8. Corren J, Lemanske RF, Hanania NA, et al.Lebrikizumab treatment in adults with asthma. N Engl J Med. 2011;365:108898.

9. Naik PK, Bozyk PD, Bentley JK, et al. Periostin promotes fibrosis and predicts progression in patients with idiopathic pulmonary fibrosis. Am J Physiol Lung Cell Mol Physiol. 2012;303:L104656.

10. Guerrot D, Dussaule JC, Mael-Ainin M, et al. Identification of periostin as a critical marker of progression/reversal of hypertensive nephropathy. PLoS One. 2012;7:e31974

11. Sen K, Lindenmeyer MT, Gaspert A, et al. Periostin is induced in glomerular injury and expressed de novo in interstitial renal fibrosis. Am J Pathol. 2011;179:1756-67.
The Journal of Critical Care Medicine 2015;1(3) • 95

12. Mael-Ainin M, Abed A, Conway S, Dussaule JC, Chatziantoniou C. Inhibition of Periostin expression protects against the development of renal inflammation and fibrosis. J Am SocNephrol. 2014;25:1724-36.

13. Satirapoj B, Wang Y, Chamberlin MP, et al. Periostin: novel tissue and urinary biomarker of progressive renal injury induces a coordinated mesenchymal phenotype in tubular cells. Nephrol Dial Transplant.2012;27:2702-11.

14. Li L, Fan D, Wang $C$, et al. Angiotensin II increases periostin expression via Ras/p38 MAPK/CREB and ERK1/2/TGF- 31 pathways in cardiac fibroblasts. CardiovascRes. 2011;91:80-89.

15. Uchida M, Shiraishi $H$, Ohta S, et al. Periostin, a matricellularprotein, plays a role in the induction of chemokines in pulmonaryfibrosis. Am J RespirCell Mol Biol. 2012;46:677-86.

16. Curat C.A. and Vogel W.F., Discoidin domain receptor 1 controls growth and adhesion of mesangial cells. J Am Soc Nephrol. 2002;13:2648-2656.

17. Flamant $M$, Placier $S$, Rodenas $A$, et al. Discoidin domain receptor 1 null mice are protected against hypertensioninduced renal disease. J Am Soc Nephrol. 2006;17:3374-81.

18. Kerroch M, Guerrot D, Vandermeersch S, et al.Genetic inhibition of discoidin domain receptor 1 protects mice against crescentic glomerulonephritis. Faseb J. 2012;26:4079-91.

19. Guerrot D, Kerroch M, Placier S, et al. Discoidin domain receptor 1 is a major mediator of inflammation and fibrosis in obstructive nephropathy. Am J Pathol. 2011;179:83-91.

20. Gross O, Girgert R, Beirowski B, et al.Loss of collagen-receptor DDR1 delaysrenalfibrosis in hereditary type IV collagendisease. Matrix Biol.2010;29:346-56.

21. Avivi-Green C, Singal M, Vogel WF. Discoidin domain receptor 1-deficient mice are resistant to bleomycin-induced lung fibrosis. Am J RespirCrit Care Med. 2006;174:420-27. 